\title{
Analysis of Prey-Predator Model in Chemostat When the Predator Produces Inhibitor
}

\section{Moniem AA*}

University College in Al-Jamoum, Umm Al-Qura University, Mecca, Saudi Arabia

\begin{abstract}
In this work, a prey-predator model in chemostat is considered when the predator produces inhibitor. This inhibitor is lethal to the prey by results in decrease of growth rate of the predator at some cost to its reproductive abilities. A Lyapunov function in the study of the global stability of a predator-free steady state is analysed. Local and global stability of other steady states, persistence analysis, as well as numerical simulations are also presented.
\end{abstract}

Keywords: Persistence; Inhibitor; Chemostat; Prey; Predator; Lyapunov function

\section{Introduction}

The chemostat is one of the standard models of an open system in ecology [1-5]. The monograph of Hsu and Waltman has various mathematical methods for analyzing chemostat models [4]. So, it is quite natural that it should be used as a model for studying detoxification problems. Many authors have studied those models [6-12]. Recently, the inhibitor has been introduced in the models for prey - predator in chemostat when the prey produces unaffected inhibitor which is lethal to neither predator nor nutrient [1]. Moniem [2] has considered a model of simple food chain in chemostat when the predator produces unaffected inhibitor which is lethal to neither prey nor nutrient.

In this paper, we consider a prey-predator model in chemo stat when the predator produces inhibitor. This inhibitor is lethal to the prey by results in decrease of growth rate of the predator at some cost to its reproductive abilities.

This paper is organized as follows: In the next section, the model is presented and some simplifications are achieved. Section 3 deals with the existence and local stability of steady states. In section 4 , we shall provide global analysis, including global stability of the boundary steady states and persistence analysis. Discussion, comments and numerical simulation are found in final section.

\section{The Model}

The interested equations are

$$
\begin{aligned}
& s^{\prime}(t)=\left(s^{0}-s(t)\right) D-\frac{1}{\gamma_{1}} f_{1}(s(t)) x(t), \\
& x^{\prime}(t)=x(t)\left(f_{1}(s(t))-D-\gamma p\right)-\frac{1}{\gamma_{2}} f_{2}(x(t)) y(t), \\
& y^{\prime}(t)=y(t)\left((1-k) f_{2}(x(t))-D\right), \\
& p^{\prime}(t)=k y(t) f_{2}(x(t))-D p, \\
& 0<s(0), \quad 0<x(o), \quad 0<y(0), \quad 0<p(0) .
\end{aligned}
$$

Where $s(t), x(t), y(t)$ and $p(t)$ are the concentration of the nutrient, prey, predator and inhibitor at time $t$ respectively. $s^{\circ}$ denotes the input concentration of the nutrient, $\mathrm{D}$ denotes the washout rate, and the parameter $\gamma$ represents the coefficient of the interaction between the inhibitor and the prey. $\gamma_{i}, \mathrm{i}=1,2$ are the Yield constants. The constant $\mathrm{k} \in(0,1)$ represents the fraction of potential growth devoted to producing the inhibitor [3].
Also we have $f_{1}(s(t))=\frac{m_{1} s(t)}{a_{1}+s(t)}$ and $f_{2}(x(t))=\frac{m_{2} x(t)}{a_{2}+x(t)}$, where $\mathrm{m}_{\mathrm{i}}, \mathrm{i}=1,2$ are the maximal growth rates, and $\mathrm{a}_{\mathrm{i}}, \mathrm{i}=1,2$ are the MichaelsMenten constants.

Now to perform the usual scaling for the chemostat, let

$$
\begin{aligned}
& \bar{s}=\frac{s}{s^{0}}, \quad \bar{x}=\frac{x}{\gamma_{1} s^{0}}, \quad \bar{y}=\frac{y}{\gamma_{1} \gamma_{2} s^{0}}, \quad \bar{t}=D t, \\
& \bar{p}=\frac{p}{\gamma_{1} \gamma_{2} s^{0}}, \quad \bar{m}_{i}=\frac{m_{i}}{D}, i=1,2, \quad \bar{a}_{1}=\frac{a_{1}}{s^{0}}, \bar{a}_{2}=\frac{a_{2}}{\gamma_{1} s^{0}} .
\end{aligned}
$$

Substituting in eqn. 1.1 and dropping the bars, the mathematical model will be reduced to the form

$$
\begin{aligned}
& s^{\prime}=1-s-f_{1}(s) x, \\
& x^{\prime}=x\left(f_{1}(s)-1-\gamma p\right)-f_{2}(x) y, \\
& y^{\prime}=y\left((1-k) f_{2}(x)-1\right), \\
& p^{\prime}=k y f_{2}(x)-p .
\end{aligned}
$$

\section{Existence and Local Stability}

Let $\mathrm{T}=\mathrm{s}+\mathrm{x}+\mathrm{y}+\mathrm{p}$ then we have $T^{\prime} \leq 1-T$, or $\lim _{t \rightarrow \infty} \sup T(t) \leq 1$. Since each component is non-negative, the system in eqn. 1.2 is dissipative and thus, has a compact, global attractor. To simplify in eqn. 1.2, let $z=p-\frac{k y}{1-k}$, we find that the system in eqn. 1.2 will take the form

$$
\begin{aligned}
& s^{\prime}=1-s-f_{1}(s) x, \\
& x^{\prime}=x\left(f_{1}(s)-1-\frac{\gamma k y}{(1-k)}\right)-f_{2}(x) y, \\
& y^{\prime}=y\left((1-k) f_{2}(x)-1\right) .
\end{aligned}
$$

Clearly $z(t) \rightarrow 0$ as $t \rightarrow \infty$ so the system in eqn. (2.1) may be viewed

*Corresponding author: Moniem AA, University College in Al-Jamoum, Umm Al-Qura University, Mecca, Saudi Arabia, Tel: 96612550 1000; E-mail: a_saltah@hotmail.com

Received June 15, 2017; Accepted October 03, 2017; Published October 27, 2017

Citation: Moniem AA (2017) Analysis of Prey-Predator Model in Chemostat When the Predator Produces Inhibitor. J Appl Computat Math 6: 367. doi: 10.4172/2168 9679.1000367

Copyright: @ 2017 Moniem AA. This is an open-access article distributed under the terms of the Creative Commons Attribution License, which permits unrestricted use, distribution, and reproduction in any medium, provided the original author and source are credited. 
as an asymptotically autonomous system with the following equations

$$
\begin{aligned}
& s^{\prime}=1-s-f_{1}(s) x, \\
& x^{\prime}=x\left(f_{1}(s)-1-\frac{\gamma k y}{(1-k)}\right)-f_{2}(x) y, \\
& y^{\prime}=y\left((1-k) f_{2}(x)-1\right) .
\end{aligned}
$$

The equilibrium point $\mathrm{E}_{0}=(1,0,0)$ always exists. If $1<f_{1}(1)$ then there is an equilibrium of in eqn. (2.2) of the form $E_{1}=\left(\lambda_{s}, 1-\lambda_{s}, 0\right)$ where ${ }_{s}$ is the unique solution of $f_{1}\left(\lambda_{\mathrm{s}}\right)-1=0$. Similarly, if $\frac{1}{1-k}<f_{2}(1)$, there is an equilibrium of the form $E_{2}=\left(s^{*} \lambda_{s}, y^{*}\right)$ where $s^{*}$ is the unique value of $s$ such that $1-\mathrm{s}-\lambda_{\mathrm{x}} f_{1}(\mathrm{~s})=0 \quad \lambda_{x}$ is the unique solution of $(1-\mathrm{k}) \mathrm{f}_{2}(\mathrm{x})-1=0$ and $y^{*}=\frac{\lambda_{x}(1-k)\left(f_{1}\left(s^{*}\right)-1\right)}{\left(1+k \lambda_{x} \gamma\right)}$.

Since the limit plane in (eqn. 2.2) is $\sum: s+y+z=1$, then by dropping $S$ equation, the system of equations eqn. (2.2) will be reduced to the form

$$
\begin{aligned}
& x^{\prime}=x\left(f_{1}(1-x-y)-1-\frac{\gamma k y}{(1-k)}\right)-f_{2}(x) y, \\
& y^{\prime}=y\left((1-k) f_{2}(x)-1\right) .
\end{aligned}
$$

It is easy to show that in eqn. 2.3 in positive plane. As a consequence, the global attractor in eqn. (2.1) lies in the set $\mathrm{z}=0$ and $\sum$ plane where in eqn. (2.3) is satisfied. When the analysis of in eqn. (2.3) is completed in this paper, the work of Thieme (11), relates the corresponding dynamics in eqns. (2.1) and (2.3), and hence in eqn. (1.2). We will show that all solutions in eqn. (2.3) tend to rest points and hence, using Thieme (10), we can find the rest points of the system in eqn. (1.2).

We now discuss the existence of steady state. The washout steady state $\mathrm{E}_{0}$ always exists. A predator-free steady state $\mathrm{E}_{1}$ exists when $\lambda_{\mathrm{s}}<1$. For the interior steady state $\mathrm{E}_{2}$ exists when ${ }_{\mathrm{s}}<1$ and ${ }_{\mathrm{s}}+\lambda_{\mathrm{x}}<1$. Note that $\mathrm{H}(\mathrm{s})=1-\mathrm{s}-\lambda_{\mathrm{x}} f_{1}(\mathrm{~s})$ is decreasing function in $\mathrm{s}$ with $0<\mathrm{H}(0)=1, \mathrm{H}\left(\mathrm{s}^{*}\right)=0$ and $\mathrm{H}(\mathrm{s})=1-\lambda_{\mathrm{s}}-\lambda_{\mathrm{x}}$ So $\lambda_{\mathrm{s}}<\mathrm{s}^{*}$.If and only if $\lambda_{\mathrm{s}}+\lambda_{\mathrm{x}}<1$.

Next theorem will discuss the local stability of this steady state by finding the eigenvalues of the associated variation matrices.

\section{Theorem 1}

If $1<\lambda_{\mathrm{S}}$ then only $\mathrm{E}_{0}$ exists and $\mathrm{E}_{0}$ is locally asymptotically stable. If $\lambda_{\mathrm{s}}<1$ and $1<\lambda_{\mathrm{s}}+\lambda_{\mathrm{x}}$ then only $\mathrm{E}_{0}$ and $\mathrm{E}_{1}$ exist, $\mathrm{E}_{0}$ is unstable, and $\mathrm{E}_{1}$ is locally asymptotically stable. If $\lambda_{\mathrm{s}}<1$ and $\lambda_{\mathrm{s}}+\lambda_{\mathrm{x}}<1$ then $\mathrm{E}_{0}, \mathrm{E}_{1}, \mathrm{E}_{2}$ exist, $\mathrm{E}_{0}$, $\mathrm{E}_{1}$ are unstable and $\mathrm{E}_{2}$ is locally asymptotically stable if

$$
\lambda_{x} f_{1}^{\prime}\left(s^{*}\right)>\left[1-\frac{\lambda_{x}\left(f_{1}\left(s^{*}\right)-1\right)}{1+k \lambda_{x} \gamma}\left(k \gamma+(1-k) f_{2}^{\prime}\left(\lambda_{x}\right)\right]\left[f_{1}\left(s^{*}\right)-1\right]\right.
$$

and consequently by an application of the Poincare-Bendixson theorem there is a periodic solution in $\Sigma$.

Proof: The Jacobian matrix in eqn. (2.3) is taken the form

$$
\left[\begin{array}{cc}
-1-y f_{2}^{\prime}(x)+f_{1}(s)-x f_{1}^{\prime}(s)-\frac{k y \gamma}{(1-k)} & -x f_{1}^{\prime}(s)-f_{2}(x)-\frac{k x \gamma}{(1-k)} \\
y(1-k) f_{2}^{\prime}(x) & (1-k) f_{2}(x)-1
\end{array}\right] .
$$

At $(1,0,0)$ this is

$$
\left[\begin{array}{ccc}
-1+f_{1}(1) & 0 & \\
0 & & -1
\end{array}\right] \text {. }
$$

The eigenvalues are on the diagonal and the washout steady state will be locally asymptotically stable if and only if $f_{1}-1<0$ or $1<\mathrm{s}$.

At $\left(\lambda_{\mathrm{S}},\left(1-\lambda_{\mathrm{S}}\right), 0\right)$ the Jacobian matrix becomes

$$
\left[\begin{array}{cc}
-\left(1-\lambda_{s}\right) f_{1}^{\prime}\left(\lambda_{s}\right) & -\left(1-\lambda_{s}\right) f_{1}^{\prime}\left(\lambda_{s}\right)-f_{2}\left(\left(1-\lambda_{s}\right)\right)-\frac{\left(1-\lambda_{s}\right) k \gamma}{(1-k)} \\
0 & (1-k) f_{2}\left(\left(1-\lambda_{s}\right)\right)-1
\end{array}\right]
$$

The two eigenvalues are $-\left(1-\lambda_{s}\right) f_{1}^{\prime}\left(\lambda_{s}\right)$ and $\left.(1-\mathrm{k})\left(1-f_{2}\right)\right)-1$ Therefore the predator - free steady state is asymptotically stable if and only if $\left.(1-\mathrm{k}) f_{2}\left(1-\lambda_{\mathrm{S}}\right)\right)-1<0$ or $1<_{\mathrm{S}}+\lambda_{\mathrm{x}}$

At $\mathrm{E}_{2}$ the Jacobian matrix takes the form

$$
\left[\begin{array}{cc}
-\lambda_{x} f_{1}^{\prime}\left(s^{*}\right)+f_{1}\left(s^{*}\right)-1-y^{*} f_{2}^{\prime}\left(\lambda_{x}\right)-\frac{k y^{*} \gamma}{(1-k)} & -\lambda_{x}\left[f_{1}^{\prime}\left(s^{*}\right)+\frac{k \gamma}{(1-k)}\right]-f_{2}\left(\lambda_{x}\right) \\
y^{*}(1-k) f_{2}^{\prime}\left(\lambda_{x}\right) & 0
\end{array}\right] .
$$

Then $\mathrm{E}_{2}$ is locally asymptotically stable if the determinant of this matrix is positive and its trace is negative, it means that

$$
\left[1-\frac{(f(s) 1)}{\lambda \gamma}\left(k \gamma+(1-k) f_{2}^{\prime}\left(\lambda_{x}\right)\right]\left[f_{1}\left(s^{*}\right)-1\right]<\lambda_{x} f_{1}^{\prime}\left(s^{*}\right) .\right.
$$

\section{Global Analysis}

\section{Theorem 2}

For $1<\lambda_{S}$ and for large $t$ all solutions in eqn. 2.2 tends to $E_{0}$.

Proof: For $1<\lambda_{\mathrm{s}}$ and for large $\mathrm{t}$ we get $\mathrm{s}(\mathrm{t})<1$ and $f_{1}(1)<1$ Therefore, the second equation 2.2 gives $x(t)<e^{-\left(1-f_{1}(1)\right) t}$, which imply $\lim _{t \rightarrow \infty} x(t)=0$. The third eqn. (2.2) becomes $y=e^{-t}$, which leads to $\lim _{t \rightarrow \infty} y(t)=0$. The first equation (2.2) has a solution $s=1+$ (constant) $\mathrm{e}^{-\mathrm{t}} \rightarrow 1$ as $\mathrm{t} \rightarrow \infty$.

\section{Theorem 3}

If $\lambda_{\mathrm{S}}<1,1<\lambda_{\mathrm{S}}+\lambda_{\mathrm{x}}$ and for large $\mathrm{t}$ then all solutions in eqn. 2.2 tend to $E_{1}$

\section{Proof}

Let

$\eta=1+\left(\frac{(1-k) f_{2}(x)+k x \gamma}{(1-k) x}\right) \frac{\left(1-\lambda_{s}-x\right)}{\left(1-(1-k) f_{2}(x)\right)}, \quad$ for $0<x \leq 1-\lambda_{s}$,

and

$$
\beta=\frac{\eta(1-k)}{\left[(1-k) f_{2}(x)+k \gamma\right]}\left[(1-k) f_{2}(x)-1\right] \quad \text { for } \quad \lambda_{x} \leq x .
$$

Let $\mathrm{C}(\mathrm{u})$ be a continuously differentiable function and $C^{\prime}(u)$ be defined by

$$
C^{\prime}(u)= \begin{cases}0 & \text { if } u \leq 1-\lambda_{s}, \\ \beta \frac{\left(u+\lambda_{s}-1\right)}{\left(\lambda_{s}+\lambda_{x}-1\right)} & \text { if } 1-\lambda_{s}<u<\lambda_{x}, \\ \beta & \text { if } \lambda_{x} \leq u .\end{cases}
$$

Note that $C^{\prime}(u)$ is linear on $\left[1-\lambda_{\mathrm{s}}, \lambda_{\mathrm{x}}\right]$ We may construct a Lyapunov function as follows:

$$
V(s, x, y)=\int_{\lambda_{s}}^{s} \frac{\left(1-\lambda_{s}\right)\left(f_{1}(\xi)-1\right)}{(1-\xi)} d \xi+x-{ }^{*} \ln (x)+\eta y+C(x) .
$$

on the set $\Psi=\{(\mathrm{s}, \mathrm{x}, \mathrm{y}): 0<\mathrm{s}+\mathrm{x}+\mathrm{y}<1\}$ where $\mathrm{x}=1-\lambda_{\mathrm{s}}$

Differentiate in eqn. 3.4 with respect to time $t$, we obtain

$$
\begin{aligned}
\dot{V} & =x\left(f_{1}(s)-1\right)\left[1+C^{\prime}(x)-\frac{\left(1-\lambda_{s}\right) f_{1}(s)}{(1-s)}\right] \\
& +y\left[\left(\frac{(1-k) f_{2}(x)+k x \gamma}{x(1-k)}\right)\left(1-\lambda_{s}-x\right)+\eta\left[(1-k) f_{2}(x)-1\right]-\left(f_{2}(x)+\frac{k \gamma}{(1-k)}\right) C^{\prime}(x)\right] .
\end{aligned}
$$

The term $x\left(f_{1}(s)-1\right)\left[1-\frac{\left(1-\lambda_{s}\right) f_{1}(s)}{(1-s)}\right]$ is non-positive for $0<\mathrm{s}<1$ and equal zero for $\mathrm{s} \in[0,1)$ if and only if $\mathrm{s}=\lambda_{\mathrm{S}}$ or $\mathrm{x}=0$ Since $C^{\prime}(x)=0$ for 
$\lambda_{s} \leq s$ and $C^{\prime}(u) \geq 0$ for $u \geq 0$, then the term $x\left(f_{1}(s)-1\right) C^{\prime}(x)$ is nonpositive for $s \in[0,1)$.

Define

$h(s, x, y)=\left[\left(\frac{f_{2}(x)}{x}+\frac{k \gamma}{(1-k)}\right)\left(1-\lambda_{s}-x\right)+\eta\left[(1-k) f_{2}(x)-1\right]-\left(f_{2}(x)+\frac{k \gamma}{(1-k)}\right) C^{\prime}(x)\right]$.

If $0<x \leq 1-\lambda_{s}$, then $\left[(1-k) f_{2}(x)-1\right] \leq 0, \quad 0 \leq\left(\frac{f_{2}(x)}{x}+\frac{k \gamma}{(1-k)}\right)\left(1-\lambda_{s}-x\right)$

and $0 \leq\left(f_{2}(x)+\frac{k \gamma}{(1-k)}\right) C^{\prime}(x)$.

Using the definition of $\eta$ we find that $h(s, x, y) \leq 0$. If $1-\lambda_{s}<\mathrm{x}<\lambda_{x}$ then all terms of $\mathrm{h}(\mathrm{s}, \mathrm{x}, \mathrm{y})$ are non-positive. If $\lambda_{x} \leq x$, then $C^{\prime}(x)=\beta$ and making use of definition of $\beta$ and $\eta$ we find that $h(s, x, y)$ will be non-positive and the second term of $\mathrm{V}$ vanishes at $\mathrm{y}=0$ therefore $\mathrm{V}$ is non-positive on $\Psi$.

Let $M$ be the largest invariant subset of $\varphi=\{(s, x, y) \in \Psi: V=0\}$ such that $\mathrm{V}=0$ at $\mathrm{S}=\lambda_{\mathrm{s}}$ or $\mathrm{x}=0$ and $\mathrm{y}=0$. More further, $\mathrm{V}$ is bounded above, any point of the form $(\mathrm{s}, 0,0)$ cannot be in the limit set of any solution initiating in the interior of $R_{+}^{3}$. $\left(\lambda_{s}, \mathrm{x}, 0\right) \mathrm{M}$ implies that $\mathrm{S}=$ and from the first equation (2.2), we get $\mathrm{x}=1-\lambda_{\mathrm{s}}$

Therefore $\mathrm{M}=\left\{\mathrm{E}_{1}\right\}$. This completes the proof.

\section{Theorem 4}

If $\lambda_{\mathrm{s}}<1$ and $\lambda_{\mathrm{s}}+\lambda_{\mathrm{x}}<1$ then the system in eqn. (2.2) is uniformly persistence.

\section{Proof}

Let $\mathrm{Z}_{1}=\{(\mathrm{s}, \mathrm{x}, \mathrm{y}): \mathrm{s} \in[0,1], \mathrm{x}, \mathrm{y} \in(0,1]\}$,

$\mathrm{Z}_{2}$ represents sx-plane : $0 \leq s, x \leq 1$,

$\mathrm{Z}_{3}$ represents sy-plane : $0 \leq s, y \leq 1$, and

$\mathrm{Z}=\mathrm{Z}_{2} \cup \mathrm{Z}_{3}$

We want to show that $Z$ is a uniformly strong repeller for $Z_{1}$ Since $\mathrm{E}_{0}$ and $\mathrm{E}_{1}$ are the only steady states in $\mathrm{Z}$. $\mathrm{E}_{0}$ is saddle in $\mathrm{R}^{3}$ and its stable manifold is $\{(s, 0, y): 0 \leq y\}$. Also, $\mathrm{E}_{1}$ is saddle in $\mathrm{R}^{3}$ and its stable manifold is $\{(s, x, 0): 0<x\}$. Then, they are weak reppelers for $\mathrm{Z}_{1}$ The stable manifold structures of $\mathrm{E}_{0}$ and $\mathrm{E}_{1}$ imply that they are not cyclically chained to each other on the boundary $\mathrm{Z}$. Therefore $\mathrm{Z}$ is a uniform strong repeller for $Z_{1}$ (see proposition in eqn.(1.2) of Thieme [12]).

So, there are $\varepsilon_{1}>0$ and $\varepsilon_{2}>0$. such that $\liminf _{t \rightarrow \infty} x(t)>\varepsilon_{1}$ and $\liminf _{t \rightarrow \infty} y(t)>\varepsilon_{2}$ where ${ }_{1}$ and $\varepsilon_{2}$ are not depending on the initial values in $\mathrm{Z}_{1}$. By using (Thieme [12]), the first eqn. (2.2) yields that there is $\varepsilon_{3}>0$ such that $\liminf _{t \rightarrow \infty} s(t)>\varepsilon_{3}$ where ${ }_{3}$ is not depending on the initial values in $\mathrm{Z}_{1}$ Proof is completed.

\section{Conclusion and Numerical Simulation}

In this paper, we consider a prey-predator model in chemostat when the predator produces inhibitor. This inhibitor is lethal to the prey by results in decrease of growth rate of the predator at some cost to its reproductive abilities. We found that the washout steady state is the global attractor, if it is the only steady state and $\lambda_{\mathrm{s}}>1$. When the washout and the predator free steady states are the only steady states, we found that $\mathrm{E}_{0}$ is unstable and $E_{1}$ is locally asymptotically stable. $\mathrm{E}_{1}$ is global attractor by constructing a Lyapunov function under condition that $\lambda_{s}<1$ and $\lambda_{s}+\lambda_{x}>1$. We also showed that $\mathrm{E}_{2}$ exists in the sense that the system is uniformly persistent and $\mathrm{E}_{2}$ is locally asymptotically stable if the determinant of this matrix is positive and its trace is negative, it means that

$$
\left[1-\frac{\lambda_{x}\left(f_{1}\left(s^{*}\right)-1\right)}{1+k \lambda_{x} \gamma}\left(k \gamma+(1-k) f_{2}^{\prime}\left(\lambda_{x}\right)\right]\left[f_{1}\left(s^{*}\right)-1\right]<\lambda_{x} f_{1}^{\prime}\left(s^{*}\right) .\right.
$$

We find by numerical simulation that eight iterative examples are presented here to show the influence of increasing the parameter $k$ on the dynamical behaviour. In all examples, parameters values in eqn. (2.2) are as follows [2]:

$$
(\mathrm{s}(0), \mathrm{x}(0), \mathrm{y}(0))=(0.1,0.7,0.8), \mathrm{m}_{1}=4.0, \mathrm{~m}_{2}=5.0, \mathrm{a}_{1}=0.6, \mathrm{a}_{2}=0.5, \gamma=0.2
$$

And we deduce that when $\mathrm{k} \in[0,0.4]$ the solution appears to approach a periodic solution. So, $\mathrm{E}_{0}, \mathrm{E}_{1}$ and $\mathrm{E}_{2}$ lose their stability (Figures 1-3). Those oscillatory solutions appear to be the results of
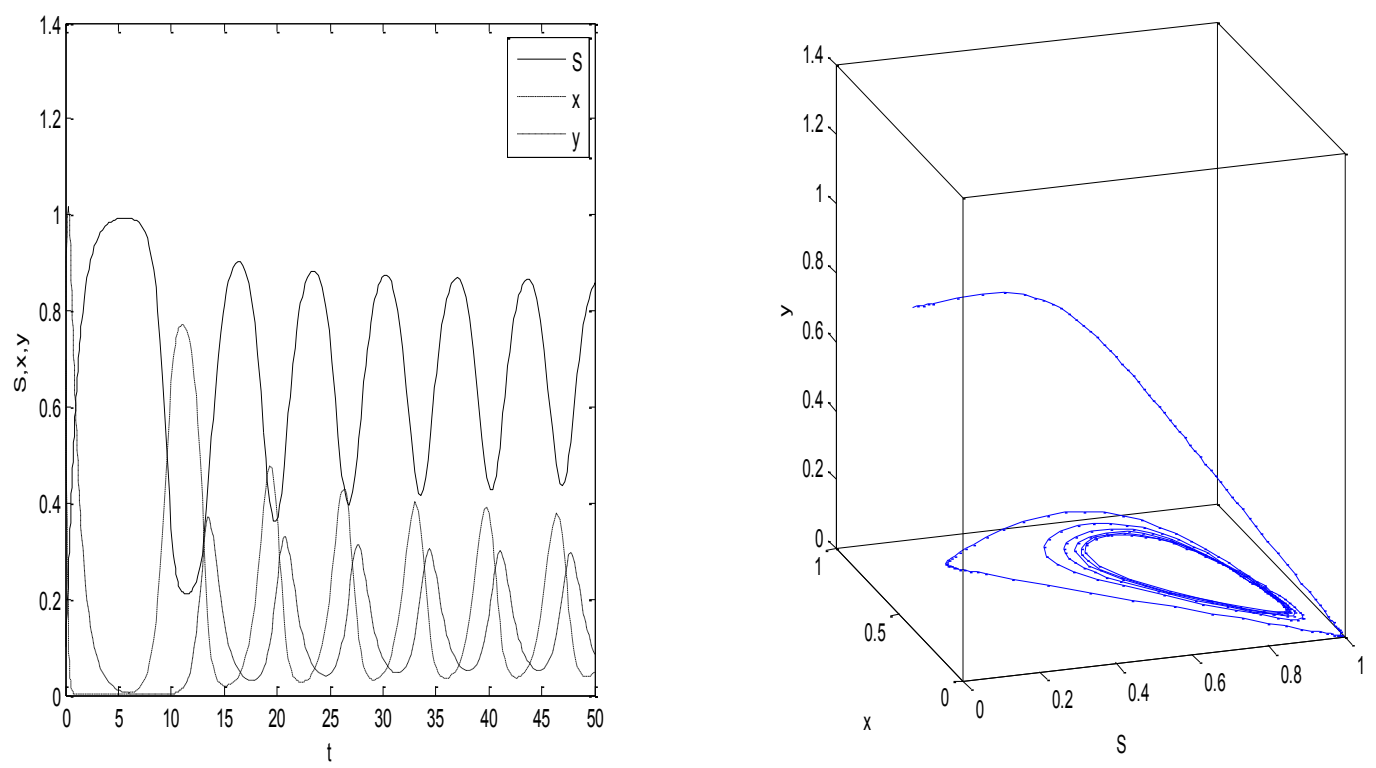

Figure 1: The results of Hopf bifurcations and oscillatory solutions are k=0.1. 

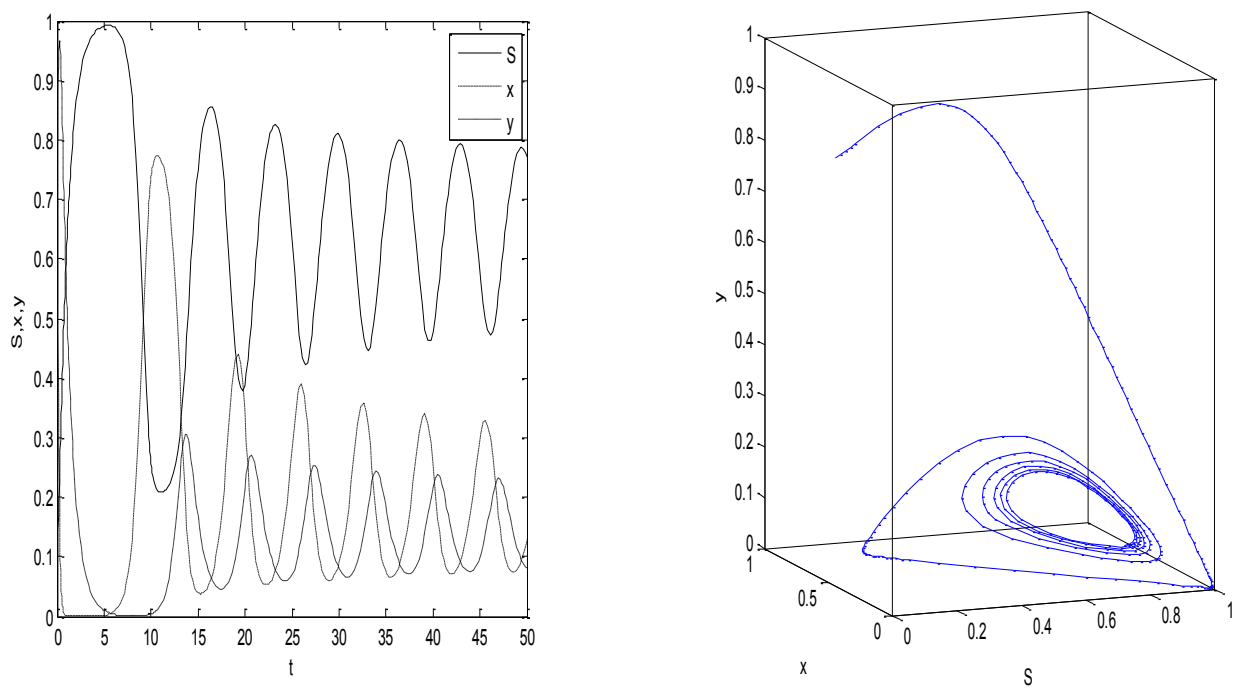

Figure 2: The results of Hopf bifurcations and oscillatory solutions are $k=0.2$
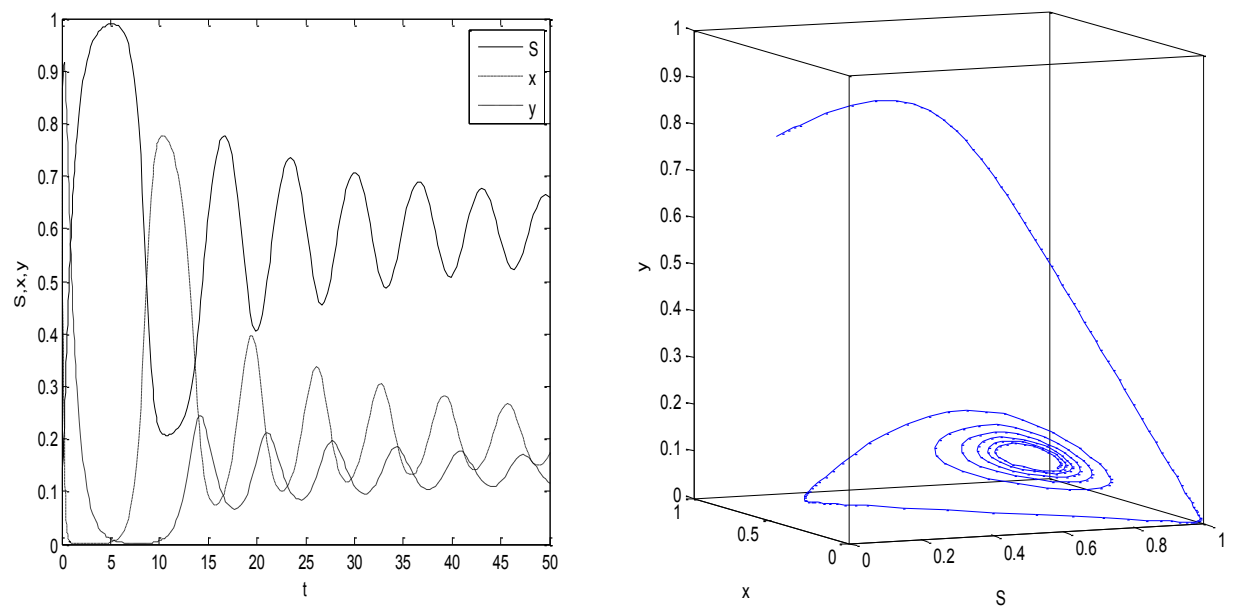

Figure 3: The results of Hopf bifurcations and oscillatory solutions oscillatory solutions are $\mathrm{k}=0.3$.
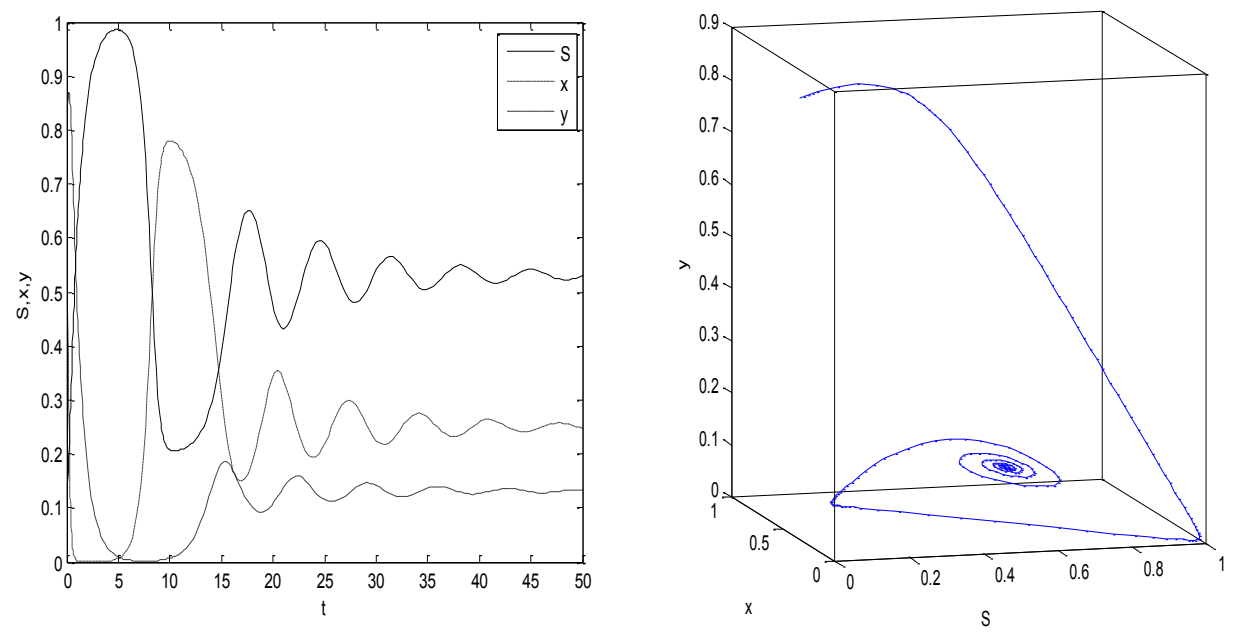

Figure 4: The solution approaches positive steady, unstable, stable states and oscillatory solutions are $\mathrm{k}=0.4$. 

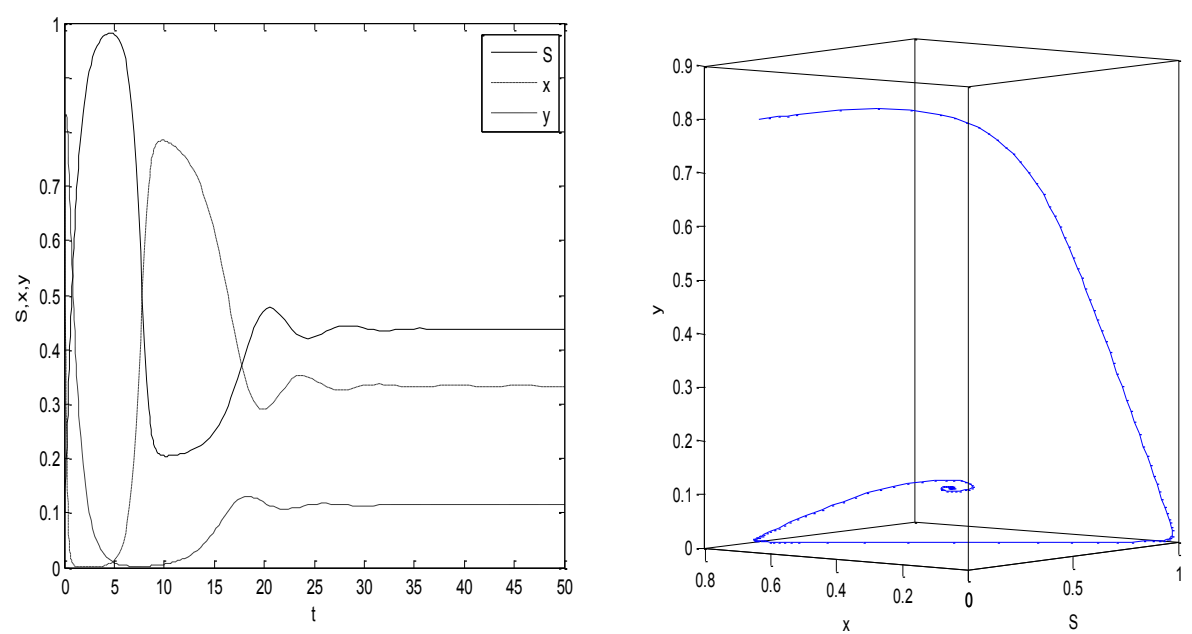

Figure 5: The solution approaches positive steady, unstable, stable states and oscillatory solutions are $k=0.5$.
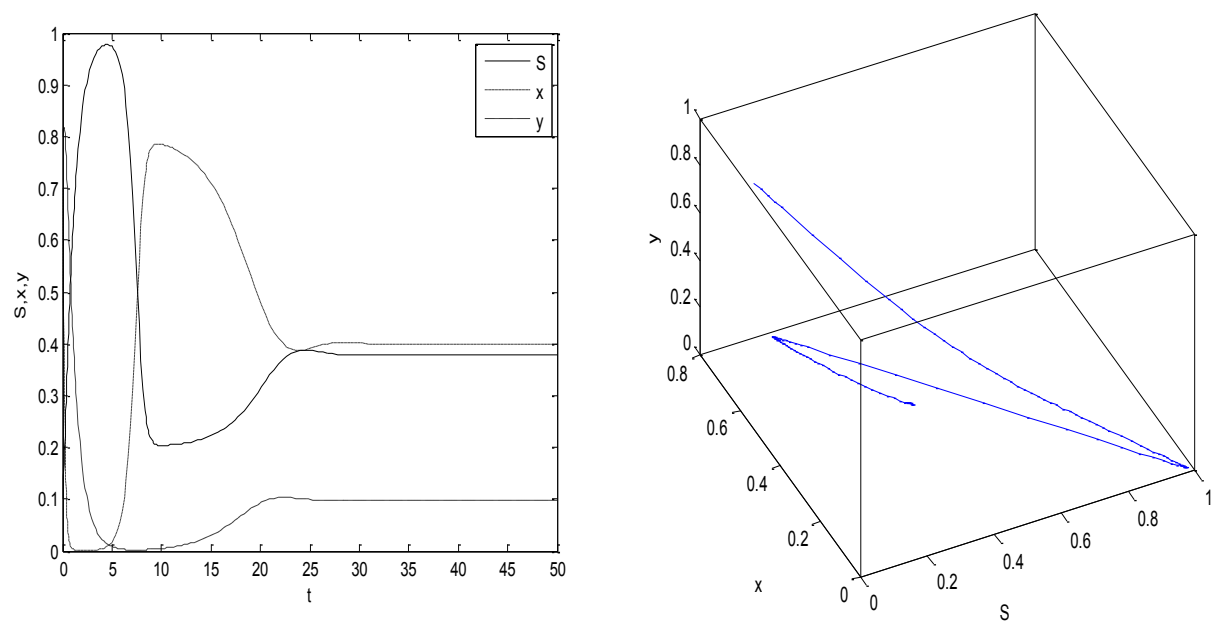

Figure 6: The solution approaches positive steady, unstable, stable states and oscillatory solutions are $\mathrm{k}=0.6$
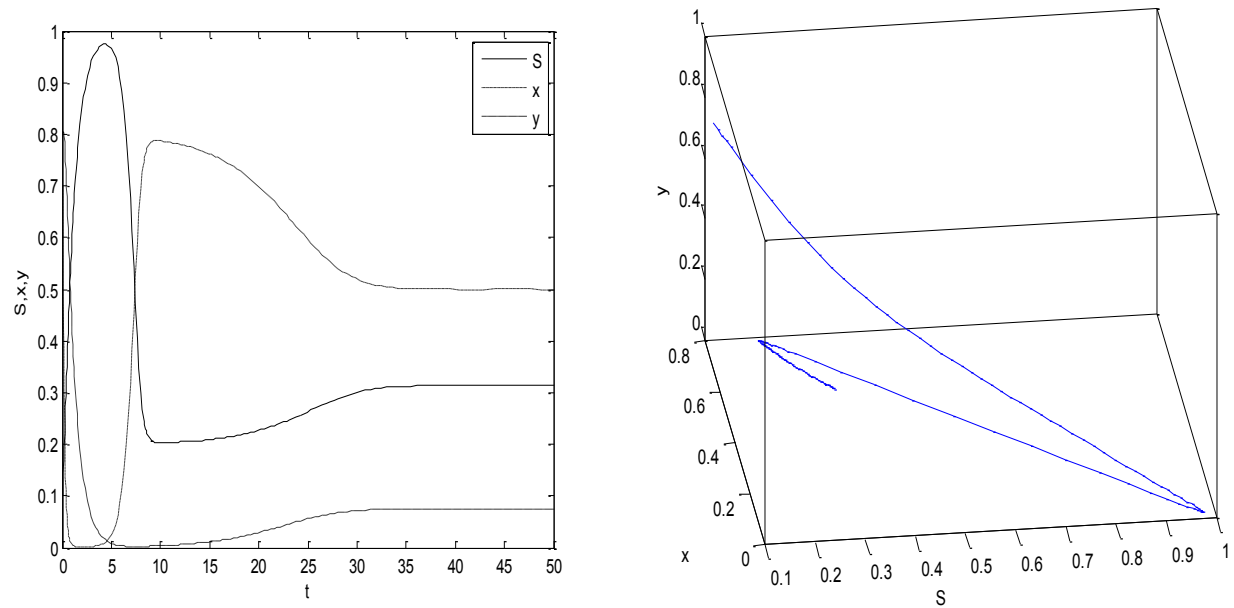

Figure 7: The solution approaches positive steady, unstable, stable states and oscillatory solutions are $\mathrm{k}=0.8$ 

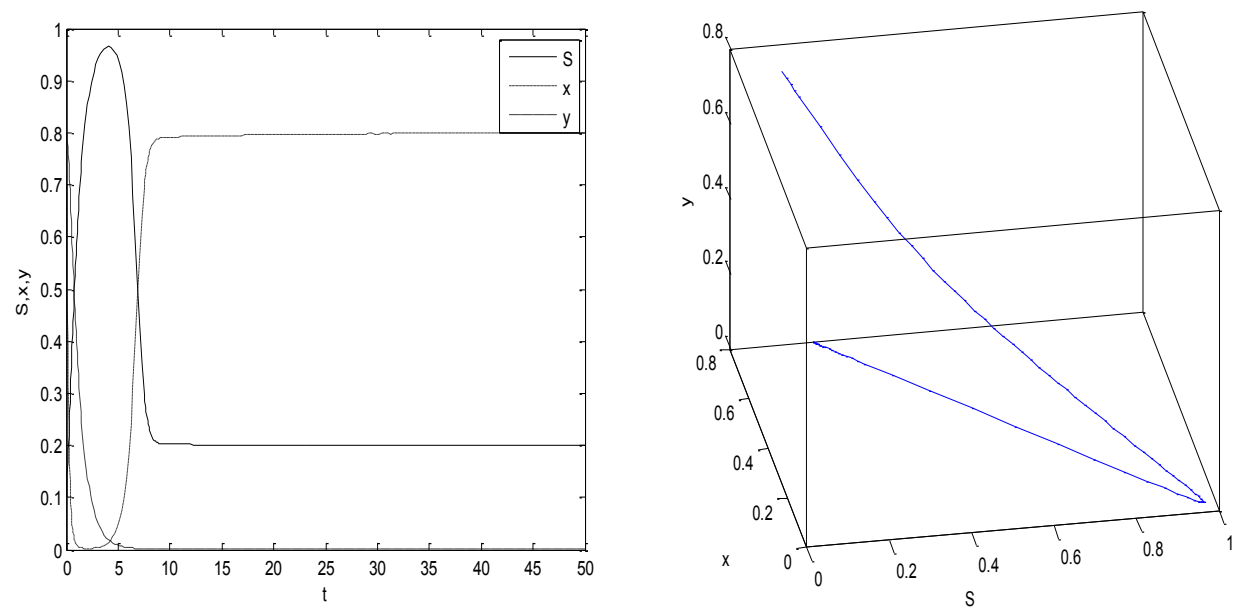

Figure 8: The solution approaches the predator-free steady, unstable, stable states and oscillatory solutions $\mathrm{k}=0.7$

Hopf bifurcations. The numerical simulation shows that the system in eqn. (2.2) has an attracting limit cycle.

Also, at $\mathrm{k} \in[0.4,6.5]$ the solution approaches a positive steady state. Both $\mathrm{E}_{0}$ and $\mathrm{E}_{1}$ are unstable and $\mathrm{E}_{2}$ is globally asymptotically stable (Figures 4-7).

For $\mathrm{k} \in[6.5,1]$ the solution approaches the predator-free steady state. $\mathrm{E}_{0}$ is unstable and $\mathrm{E}_{1}$ is globally asymptotically stable (Figure 8). All left figures plot in time courses and all right figurers plot the trajectory in $(\mathrm{s}, \mathrm{x}, \mathrm{y})$ space. We also, deduce that no difference between this paper and Moniem [2] as numerical simulation, even $\gamma$ changes its value on interval $[0,1$ [for each value of $\mathrm{k}$.

\section{References}

1. Moniem AA (2012) "Simple Food Chain in Chemostat When the Predator Produces Unaffected Toxin". $6^{\text {th }}$ International Conference on Mathematics and Engineering Physics, pp 29-31.

2. Moniem AA (2012) "Prey-Predator in Chemostat When the Prey Produces Unaffected Inhibitor". 6 th International Conference on Mathematics and Engineering Physics, pp: 29-31.

3. Braselton JP, Waltman P (2001) A Competition Model With Dynamically Allocated Inhibitor Production. Mathematical Biosciences 173: 55-84.

4. Hsu SB, Waltman P (1992) Analysis of a Model Of Two Competitors In a
Chemostat with an External Inhibitor. Journal on Applied Mathematics 52: 528-540.

5. Hsu SB, Waltman P (1998) Competition In The Chemostat When One Competitor Produces A Toxin. Japan Journal of Industrial and Applied Mathematics 15: 471-490.

6. Hsu SB, Luo Tk, Waltman P (1995) Competition between Plasmid-Bearing and Plasmid-Free Organisms in a Chemostat with an Inhibitor. Journal of Mathematical Biology 34: 225-238.

7. Hsu SB, Li YS, Waltman P (2000) Competition in the Presence of a Lethal External Inhibitor. Mathematical Biosciences 176: 177-199.

8. Lenski RE, Hattingh S (1986) Coexistence of two Competitors on one Resource and one Inhibitor; A Chemostat Model based on Bacteria and Antibiotics. Journal of Theoretical Biology 122: 83-93.

9. Li J, Feng Z, Zhang J, Lou J (2006) A Competition Model of the Chemostat with an External Inhibitor. Mathematical Biosciences and Engineering 3: 111-123.

10. Smith H, Waltman P (1995) The Theory of Chemostat. Cambridge University Press.

11. Thieme HR (1992) Convergence Results and a Poincare - Bendixson Trichotomy for Asymptotically Autonomous Differential Equations. Journal of Mathematical Biology 30: 755-763.

12. Thieme HR (1992) Persistence Under Relaxed Point - Dissipativeity (With Applications To An Epidemic Model). SIAM Journal on Mathematical Analysis 24: $407-435$ 